PROCEEDINGS OF THE

AMERICAN MATHEMATICAL SOCIETY

Volume 129, Number 2, Pages 573-580

S 0002-9939(00)05603-3

Article electronically published on July 27, 2000

\title{
THE BERNSTEIN PROBLEM FOR COMPLETE LAGRANGIAN STATIONARY SURFACES
}

\author{
CHIKAKO MESE
}

(Communicated by Bennett Chow)

\begin{abstract}
In this paper, we investigate the global geometric behavior of lagrangian stationary surfaces which are lagrangian surfaces whose area is critical with respect to lagrangian variations. We find that if a complete oriented immersed lagrangian surface has quadratic area growth, one end and finite topological type, then it is minimal and hence holomorphic. The key to the proof is the mean curvature estimate of Schoen and Wolfson combined with the observation that a complete immersed surface of quadratic area growth, finite topology and $L^{2}$ mean curvature has finite total absolute curvature.
\end{abstract}

\section{INTRODUCTION}

In $[\mathrm{SW}$, R. Schoen and J. Wolfson introduces the constrained variational problem of extremizing the volume amongst submanifolds of $\mathbf{R}^{2 n}$ which are lagrangian and spanning a given boundary. This study of the "Lagrangian Plateau Problem" is motivated by issues arising in nonlinear elasticity and mirror symmetry. (See [SW] for references.) There, many interesting analogues of the classical Plateau Problem are discussed. As in the classical minimal surface theory, it is natural to pass from the local theory to the study of the global geometric behavior. In this paper, we study complete surfaces for which the area of any compact subset is critical for arbitrary compactly supported variations through lagrangian surfaces. We have the following result.

Theorem 1. A complete oriented immersed lagrangian surface $\iota: \Sigma \rightarrow \mathbf{R}^{4}$ with quadratic area growth, one end and finite topological type is lagrangian stationary if and only if it is stationary.

We note that a lagrangian stationary surface with boundary is not necessarily stationary. The above theorem has the following corollary.

Corollary 2. A complete oriented immersed lagrangian stationary surface $\iota: \Sigma \rightarrow$ $\mathbf{R}^{4}$ of quadratic area growth, one end and finite topological type is holomorphic (with respect to the complex structure $\tilde{J}$ defined below).

Received by the editors April 12, 1999.

1991 Mathematics Subject Classification. Primary 58E12; Secondary 53C15.

The author would like to thank Professor Richard Schoen for introducing her to this problem and Professor Paul Yang for his interest in this work. Additionally, she thanks Professor Francis Bonahon for many useful conversations. 
This paper is motivated by the Bernstein Theorem. The original statement, due to Bernstein in 1951, says that complete minimal graphs in $\mathbf{R}^{3}$ are planes. There has been extensive work in generalizing the Bernstein Theorem (see, for example, O1], [O2], [O3], [X], [C], BdC], [SSY], [FS], [dCP]). Most of the literature on this subject assumes that the co-dimension is 1 . A Bernstein-type theorem for surfaces of higher co-dimensional was investigated by M. Micallef $[\mathrm{M}]$.

Recall that the Euler-Lagrange equation for the area implies that a surface is stationary if and only if the mean curvature vector vanishes identically. The point of Theorem 1 is that we can prove the vanishing of the mean curvature vector of a lagrangian surface even when it is stationary with respect to a smaller class of variations of the surface, namely the competing surfaces are required to also be lagrangian.

We note that the assumption in Theorem 1 that the surface has one end is essential. The infinite cylinder $x_{1}^{2}+y_{1}^{2}=1$ in $\mathbf{R}^{4}$ with coordinates given by $\left(x_{1}, x_{2}, y_{1}, y_{2}\right)$ is lagrangian stationary but not stationary.

\section{Preliminaries}

A Riemannian manifold is a manifold $M$ with a Riemannian metric $g$. We call a submanifold $\Sigma$ stationary (or minimal) if the volume (defined by metric $g$ ) of any compact subset is critical for arbitrary compactly supported variations. Using the Euler-Lagrange equation, this property translates to the geometric property that its mean curvature vector vanishes identically.

A symplectic manifold is an even-dimensional manifold $M^{2 n}$ with a closed nondegenerate one-form $\omega$. This means that $d \omega=0$ and if $\omega(X, Y)=0$ for all tangent vectors $X$, then $Y=0$. A submanifold $\Sigma$ of dimension $n$ is said to be lagrangian if the restriction of $\omega$ vanishes identically on $\Sigma$, i.e. $\omega(X, Y)=0$ for vectors $X, Y$ tangent to $\Sigma$ at any point.

A Kähler manifold $M$ is an even dimensional manifold which has a Riemannian metric $g$ and an almost complex structure $J$ which is orthogonal with respect to $g$ (i.e. $g(J X, J Y)=g(X, Y))$ and parallel with respect to the Levi-Civita connection defined by $g$. This makes $M$ a symplectic manifold where the symplectic form $\omega$ is defined by $\omega(X, Y)=g(J X, Y)$. In such a manifold, we can consider the following variational problem: given a class of lagrangian submanifolds of $M$ (for example, with fixed boundary or homology class), find one that extremizes the volume. Deformations of a lagrangian submanifold preserving the lagrangian property are abundant and this makes the variational problem interesting. We call a lagrangian submanifold lagrangian stationary if the volume of any compact subset is critical for any compactly supported variation through lagrangian submanifolds. Lagrangian submanifolds which are stationary are special lagrangian (see [HL).

Just as in the stationary submanifolds, an important geometric property of a lagrangian stationary submanifold $\Sigma$ of $(M, \omega)$ concerns the mean curvature vector $H$. To $H$, we can associate a 1 -form $\alpha$ defined by $\alpha(\cdot)=\omega(H, \cdot)$. In a Kähler manifold, a calculation $([\mathrm{B}], \mathrm{W}])$ shows that $d \alpha=\operatorname{ric}$ where $\operatorname{ric}(X, Y)$ is a two-form associated with the Ricci curvature (i.e. $\operatorname{ric}(X, Y)=\operatorname{Ric}(J X, Y)$ ). In particular, if the manifold is Kähler-Einstein $(R i c=g)$, then $d \alpha=\omega$ and $\alpha$ is a closed oneform. By the Poincaré Lemma, $\alpha$ is locally exact and there exists a locally defined function $\beta$ so that $d \beta=\alpha$. The Euler-Lagrange equation $[\mathrm{Oh}$ implies that $\alpha$ is a 
harmonic one-form, or equivalently that $\beta$ is a harmonic function in the conformal structure induced by $g$.

We will study lagrangian stationary surfaces in Euclidean space $\mathbf{R}^{2 n}$ with the standard complex structure $J$ defined by

$$
J\left(\frac{\partial}{\partial x_{i}}\right)=\frac{\partial}{\partial y_{i}}, \quad J\left(\frac{\partial}{\partial y_{i}}\right)=-\frac{\partial}{\partial x_{i}} .
$$

The symplectic form is given by $\omega=\sum_{i=1}^{n} d x_{i} \wedge d y_{i}$. We note that if $n=2$, there is a one-to-one correspondence between special lagrangian surfaces in complex structure $J$ and complex surfaces in the complex structure $\tilde{J}$ defined by

$$
\tilde{J}\left(\frac{\partial}{\partial x_{1}}\right)=\frac{\partial}{\partial x_{2}}, \tilde{J}\left(\frac{\partial}{\partial x_{2}}\right)=-\frac{\partial}{\partial x_{1}}, \tilde{J}\left(\frac{\partial}{\partial y_{1}}\right)=\frac{\partial}{\partial y_{2}}, \tilde{J}\left(\frac{\partial}{\partial y_{2}}\right)=-\frac{\partial}{\partial y_{1}} .
$$

\section{Proof of the Main theorem}

We will utilize the fundamental result of Schoen and Wolfson. In fact, a close inspection of the proof of Theorem 1 in SW] gives us Theorem B (which is a slightly stronger statement than that in $[\mathrm{SW}]$ ). We let $\iota: \Sigma \rightarrow \mathbf{R}^{4}$ be a lagrangian immersion where $\Sigma$ is a surface of finite topological type with connected boundary $\partial \Sigma$ and where $\iota(\Sigma)$ is lagrangian stationary surface. Let $B(\rho)$ be the ball of radius $\rho$ centered at the origin and assume that $\iota(0)$ lies inside $B\left(\frac{1}{2}\right)$ and $\iota(\partial \Sigma)$ lies outside $B(1)$. Let $\Sigma_{\frac{1}{2}}$ denote the connected component of 0 in $\iota^{-1}\left(\iota(\Sigma) \bigcap B\left(\frac{1}{2}\right)\right)$.

Theorem 3 (Schoen-Wolfson). There is a constant $C$ depending only on the area of $\iota(\Sigma)$ inside the unit ball and the topology of $\Sigma$ so that

$$
\int_{\Sigma_{\frac{1}{2}}}|H|^{2} \leq C
$$

Theorem 3 is used to show the following.

Lemma 4. Let $\iota: \Sigma \rightarrow \mathbf{R}^{4}$ be a complete oriented immersed lagrangian stationary surface with quadratic area growth, one end and finite topology. Then

$$
\int_{\Sigma}|H|^{2} \leq C
$$

where $C$ depends on the topology of $\Sigma$.

Proof. Fix $p \in \Sigma$ and assume without loss of generality that $\iota(p)=0$. Let $U_{t}$, $t \rightarrow \infty$, be a compact exhaustion of $\Sigma$ with smooth boundary $\partial U_{t}$ and $p \in U_{t}$. Since $\Sigma$ has only one end, we can choose them so that $U_{t}$ 's have fixed topological type and one boundary component. Let $r(t)=\min \left\{|\iota(x)|: x \in \partial U_{t}\right\}$ and $U_{t}^{\prime}$ be the connected component containing 0 of $\iota^{-1}\left(\iota\left(U_{t}\right) \cap B\left(\frac{r(t)}{2}\right)\right)$. Since $\iota$ is an immersion of a surface with quadratic area growth, $\iota$ is a proper immersion. Hence $r(t) \rightarrow \infty$ as $t \rightarrow \infty$ and $U_{t}^{\prime}$ is also a exhaustion of $\Sigma$. Let $\iota_{t}=\frac{1}{r(t)} \iota$. By construction $\iota_{t}\left(\partial U_{t}\right)$ lies outside $B(1)$. By Theorem 3 , the scale invariance of the $L^{2}$ norm of $H$ and the fact that $\Sigma$ is of quadratic area growth, there is a constant $C$ independent of $t$ so that

$$
\int_{U_{t}^{\prime}}|H|^{2} \leq C
$$


We get the result by observing that

$$
\int_{\Sigma}|H|^{2}=\lim _{t \rightarrow \infty} \int_{U_{t}^{\prime}}|H|^{2} \leq C .
$$

Additionally, we will show that a complete oriented immersed lagrangian stationary surface of quadratic area growth with one end has finite total absolute curvature. This is accomplished by combining Lemma 4 with Theorem 5 . We note that Theorem 5 generalizes a result of $\mathrm{Q}$. Chen [Ch] who proved that a minimal surface of quadratic area growth and finite topological type has finite total curvature. P. $\mathrm{Li}$ [ $\mathrm{Li}$ ] has shown that a complete surface with quadratic area growth, finite topological type and whose curvature does not change signs outside a compact set has finite total curvature. Since minimal surfaces have nonpositive curvature, Li's result implies the theorem of Chen but it does not imply the case stated in Theorem [5] Theorem [5] is proved by modifying the arguments of [Ch].

Theorem 5. If a complete oriented immersed surface $\Sigma \subset \mathbf{R}^{k}$ of quadratic area growth and finite topology has the property that

$$
\int_{\Sigma}|H|^{2}<\infty
$$

then it has finite total absolute curvature and is conformally equivalent to a Riemann surface minus a point.

Proof. By Sard's Theorem applied to the distance function $r(x)=|x|$ on $\Sigma$, $\partial(\Sigma \cap B(t))$ is a one-dimensional submanifold of $\mathbf{R}^{k}$ for a.e. $t$. We let $O \subset \mathbf{R}$ be a set of such $t$ 's. For $t \in O$, let $\Sigma_{t}$ be a union of $\Sigma \cap \overline{B(t)}$ and the bounded components of $\Sigma-B(t)$. Since $\Sigma-\Sigma_{t}$ contains no topological disks, $\chi\left(\Sigma-\Sigma_{t}\right) \leq 0$ and hence

$$
-\chi\left(\Sigma_{t}\right)=-\chi(\Sigma)+\chi\left(\Sigma-\Sigma_{t}\right) \leq-\chi(\Sigma) .
$$

Let $H, K$ and $A$ be the mean curvature vector, Gauss curvature and second fundamental form, respectively. Since

$$
\int_{\Sigma}|K|=\lim _{t \rightarrow \infty} \int_{\Sigma_{t}}|K|
$$

we need only show that there is a constant $C_{1}$ so that

$$
\int_{\Sigma_{t}}|K| \leq C_{1}
$$

for all $t$. By the scale invariance of the above integral and the assumption of quadratic area growth, we need to only show

$$
\int_{\Sigma_{1 / 2}}|K| \leq C_{1}
$$

where $C_{1}$ is dependent only on the area of $\Sigma \bigcap B(1)$.

We will let

$$
\mathbf{K}(t)=\int_{\Sigma_{t}}-K, \mathbf{H}(t)=\int_{\Sigma_{t}}|H|^{2} \text { and } \mathbf{A}(t)=\int_{\Sigma_{t}}|A|^{2} .
$$


By slightly deforming the surface if necessary, we may assume that the set $[0,1]-O$ is finite. Hence the first variation formula of length applied to the family of curves $\left\{\partial \Sigma_{t}\right\}$ gives

$$
\frac{d}{d t} \int_{\partial \Sigma_{t}}|\nabla r|=\int_{\partial \Sigma_{t}} k_{g}+\int_{\partial \Sigma_{t}} \nabla_{v}|\nabla r|
$$

where $k_{g}$ is the geodesic curvature of $\partial \Sigma_{t}$ in $\Sigma$ and $v$ is the velocity vector with respect to the flow of $\Sigma$ which generates the curves $\partial \Sigma_{t}$. The co-area formula formula gives

$$
\int_{\partial \Sigma_{t}} \nabla_{v}|\nabla r|=\frac{d}{d t} \int_{\Sigma_{t}}|\nabla r|\left(\nabla_{v}|\nabla r|\right)=\frac{1}{2} \frac{d}{d t} \int_{\Sigma_{t}} \nabla_{v}|\nabla r|^{2}
$$

Furthermore, on $\Sigma$,

$$
\triangle r=\operatorname{div}(\nabla r)=\frac{2-|\nabla r|^{2}+\vec{x} \cdot H}{r}
$$

where $\vec{x}$ is the position vector of $\Sigma \subset \mathbf{R}^{k}$. So,

$$
\frac{d}{d t} \int_{\partial \Sigma_{t}}|\nabla r|=\frac{d}{d t} \int_{\Sigma_{t}} \Delta r=\frac{d}{d t} \int_{\Sigma_{t}} \frac{2-|\nabla r|^{2}+\vec{x} \cdot H}{r}
$$

Combining the Gauss-Bonet Theorem with the above, we obtain

$$
\frac{d}{d t} \int_{\partial \Sigma_{t}} \frac{2-|\nabla r|^{2}+\vec{x} \cdot H}{r}=\mathbf{K}(t)+2 \pi \chi\left(\Sigma_{t}\right)+\frac{1}{2} \frac{d}{d t} \int_{\Sigma_{t}} \nabla_{v}|\nabla r|^{2} .
$$

Let $1 / 2 \leq t_{0}<t_{1}<1$. First assume $t_{0}, t_{1}$ are contained in a connected component of $O$. Integrating from $t_{0}$ to $t_{1}$,

$$
\int_{t_{0}}^{t_{1}} \mathbf{K}(t) d t=\int_{\Sigma_{t_{1}}-\Sigma_{t_{0}}} \frac{2-|\nabla r|^{2}+\vec{x} \cdot H}{r}-\int_{t_{0}}^{t_{1}} 2 \pi \chi\left(\Sigma_{t}\right)-\frac{1}{2} \int_{\Sigma_{t_{1}}-\Sigma_{t_{0}}} \nabla_{v}|\nabla r|^{2} .
$$

A calculation shows

$$
\left.\left.\left|\nabla_{v}\right| \nabla r\right|^{2}|\leq 2| A|| \nabla^{\perp} r\left|+\frac{2}{r}\right| \nabla^{\perp} r\right|^{2} .
$$

Since $\left[t_{0}, t_{1}\right]$ contains only regular values of $r(x)$ by assumption, $\Sigma_{t_{1}}-\Sigma_{t_{0}}$ is contained in $B\left(t_{1}\right)$. Therefore,

$$
\int_{t_{0}}^{t_{1}} \mathbf{K}(t) d t \leq C_{2}+C_{3}\left[\left(\mathbf{H}\left(t_{1}\right)-\mathbf{H}\left(t_{0}\right)\right)^{1 / 2}+\left(\mathbf{A}\left(t_{1}\right)-\mathbf{A}\left(t_{0}\right)\right)^{1 / 2}\right]
$$

where the constants $C_{2}, C_{3}$ are only dependent on the area of $\Sigma \cap B(1)$ and the upper bound of $-\chi\left(\Sigma_{t}\right)$. 
Now assume $\{\bar{t}\}=\left[t_{0}, t_{1}\right]-O$. We then have

$$
\begin{aligned}
\int_{t_{0}}^{t_{1}} \mathbf{K}(t) d t= & \lim _{\epsilon \rightarrow 0^{+}}\left(\int_{\Sigma_{t_{1}}-\Sigma_{\bar{t}+\epsilon}}+\int_{\Sigma_{\bar{t}-\epsilon}-\Sigma_{t_{0}}} \frac{2-|\nabla r|^{2}+\vec{x} \cdot H}{r}\right) \\
& -\int_{t_{0}}^{t_{1}} 2 \pi \chi\left(\Sigma_{t}\right)+\frac{1}{2} \lim _{\epsilon \rightarrow 0^{+}}\left(\int_{\Sigma_{t_{1}}-\Sigma_{\bar{t}+\epsilon}}+\int_{\Sigma_{\bar{t}-\epsilon}-\Sigma_{t_{0}}} \nabla_{v}|\nabla r|^{2}\right) \\
\leq & C_{2}+C_{3} \lim _{\epsilon \rightarrow 0^{+}}\left(\int_{\Sigma_{t_{1}}-\Sigma_{\bar{t}+\epsilon}}+\int_{\Sigma_{\bar{t}-\epsilon}-\Sigma_{t_{0}}}|H|^{2}\right)^{1 / 2} \\
& +C_{3} \lim _{\epsilon \rightarrow 0^{+}}\left(\int_{\Sigma_{t_{1}}-\Sigma_{\bar{t}+\epsilon}}+\int_{\Sigma_{\bar{t}-\epsilon}-\Sigma_{t_{0}}}|A|^{2}\right)^{1 / 2} .
\end{aligned}
$$

But by Morse Theory, $\Sigma_{\bar{t}-\epsilon} \subset \Sigma_{\bar{t}+\epsilon}$ and hence

$$
\left(\Sigma_{t_{1}}-\Sigma_{\bar{t}+\epsilon}\right) \cup\left(\Sigma_{\bar{t}-\epsilon}-\Sigma_{t_{0}}\right) \subset \Sigma_{t_{1}}-\Sigma_{t_{0}}
$$

and equation (3) holds in this case. Repeating the above argument if necessary, we see that (3) holds for all $t_{0}, t_{1} \in[1 / 2,1]$. Using the equality

$$
|A|^{2}=-2 K+|H|^{2}
$$

we obtain

$$
\frac{1}{2} \int_{t_{0}}^{t_{1}}(\mathbf{A}(t)-\mathbf{H}(t)) d t \leq C_{2}+C_{3}\left[\left(\mathbf{H}\left(t_{1}\right)-\mathbf{H}\left(t_{0}\right)\right)^{1 / 2}+\left(\mathbf{A}\left(t_{1}\right)-\mathbf{A}\left(t_{0}\right)\right)^{1 / 2}\right]
$$

and using (11) we get

$$
\int_{t_{0}}^{t_{1}} \mathbf{A}(t) d t \leq C_{4}+2 C_{3}\left(\mathbf{A}\left(t_{1}\right)-\mathbf{A}\left(t_{0}\right)\right)^{1 / 2} .
$$

Now we can follow the iteration scheme of $\mathrm{Ch}$ to prove that this inequality implies $\mathbf{A}(t) \leq C_{5}$ where $C_{5}$ is dependent on $C_{3}$ and $C_{4}$. Since (4) implies $2|K| \leq|A|^{2}+$ $|H|^{2}$, we have proved the first assertion of Theorem [5. The second assertion of Theorem 5 follows from Huber's result $[\mathrm{H}]$ that states that, if the negative part of the Gaussian curvature is integrable, then the surface is conformally equivalent to a compact Riemann surface with finitely many punctures with each puncture corresponding to an end.

We will now prove the main theorem. A similar argument in [SW] shows that a closed lagrangian stationary submanifold has vanishing mean curvature vector.

Proof of the main theorem. By Theorem 5, a complete lagrangian surface of quadratic area growth and one end can be expressed by a conformal immersion $\iota: \Sigma \rightarrow$ $M$ where $\Sigma$ is a Riemann surface minus a point $p$. Let $H$ be the mean curvature vector of this immersion. Let $O$ be a neighborhood of the zero section in the normal bundle of $\Sigma$ where the zero section is identified with $\Sigma$. We extend $\iota$ to an immersion $I: O \rightarrow M$. I is then a local diffeomorphism and we can pull back both $g$ and $J$ to $O$ using $I$. Define a one-form on $\Sigma$ by setting $\alpha(\cdot)=\omega(H, \cdot)$. Since $\int_{\Sigma}|\alpha|^{2} d v_{g}=\int_{\Sigma}|H|^{2} d v_{g}<\infty, \alpha$ extends smoothly to 0 at $p$. Hence $\alpha$ can be seen as a closed one-form on the Riemann surface $\bar{\Sigma}$ and is locally exact by the Poincaré lemma. Let $U \subset \bar{\Sigma}$ be a coordinate neighborhood of $p$ with conformal coordinate $(x, y)$ and let $h$ be a locally defined function so that $d h=\alpha$ in $U$. Since $h$ is defined only up to a constant, we may assume $\int_{U} h=0$. We can choose a neighborhood 
$N \subset \subset U$ of $p$ and a cut-off function so that $\varphi \equiv 1$ in $U-N, \varphi \equiv 0$ in $N$ and $\|\bar{\nabla} \varphi\| \leq(2 \operatorname{diam} U)^{-1}$ where $\bar{\nabla}$ and $\|\cdot\|$ are gradient and norm with respect to the conformal coordinates (and not the metric $g$ ) and diam $U$ is given in terms of the conformal distance. Define a one-form $\bar{\alpha}$ by

$$
\bar{\alpha}=\left\{\begin{array}{cc}
\alpha & \text { in } \Sigma-U \\
d(\varphi h) & \text { in } U .
\end{array}\right.
$$

By construction, $\bar{\alpha}$ is a locally exact one-form so that $\bar{\alpha} \equiv 0$ in a neighborhood of $p$. Now define a one-form $\beta$ on $\bar{O}$ by $\beta=\Pi^{*} \bar{\alpha}$. By construction $\beta$ is locally exact. There exists a unique vector field $V$ on $O$ so that $\beta(X)=\omega(V, X)$. By construction, $V$ is locally hamiltonian. Hence if $F_{t}$ is the flow defined by $V$ near $\Sigma$, then $F_{t}$ is a symplectic transformation. This implies $F_{t}(\Sigma)$ is a lagrangian surface and $I\left(F_{t}(\Sigma)\right)$ is a lagrangian variation. Noting that $V=H$ on $\Sigma-U$, the first variation of area gives

$$
0=\int_{\Sigma} g(V, H) d v_{g}=\int_{\Sigma-U}|H|^{2} d v_{g}+\int_{U} g(\nabla(\varphi h), H) d v_{g} .
$$

Here $\nabla$ and $|\cdot|$ are the gradient and norm with respect to the metric $g$ as before. Hence,

$$
\int_{\Sigma-U}|H|^{2} d v_{g} \leq \frac{1}{2} \int_{U}|\nabla(\varphi h)|^{2} d v_{g}+\frac{1}{2} \int_{U}|H|^{2} d v_{g}
$$

By using the conformal invariance, we obtain

$$
\begin{aligned}
\int_{U}\|\bar{\nabla}(\varphi h)\|^{2} d x d y= & \int_{U}\left(\varphi^{2}\|\bar{\nabla} h\|^{2}+2 \bar{\nabla} \varphi \cdot \bar{\nabla} h+h^{2}\|\bar{\nabla} \varphi\|^{2}\right) d x d y \\
\leq & \int_{U}\left(\|\bar{\nabla} h\|^{2}+\frac{2}{\operatorname{diam} U}\|\bar{\nabla} h\|+h^{2}\|\bar{\nabla} \varphi\|^{2}\right) d x d y \\
\leq & \int_{U}\|\bar{\nabla} h\|^{2} d x d y+\frac{2(\text { Area } U)^{1 / 2}}{\operatorname{diam} U}\left(\int_{U}\|\bar{\nabla} h\|^{2} d x d y\right)^{1 / 2} \\
& \quad+\frac{1}{(\operatorname{diam} U)^{2}} \int_{U} h^{2} d x d y \\
\leq & C\left(\int_{U}\|\bar{\nabla} h\|^{2}+2\left(\int_{U}\|\bar{\nabla} h\|^{2} d x d y\right)^{1 / 2}\right) .
\end{aligned}
$$

The last inequality uses the Poincaré inequality

$$
\frac{1}{(\operatorname{diam} U)^{2}} \int_{U} h^{2} \leq C \int_{U}\|\bar{\nabla} h\|^{2} d x d y
$$

(since we can assume that $U$ is a ball in $\mathbf{R}^{2}$ ). Since $\int_{\Sigma}|H|^{2}<\infty$, by choosing $U$ sufficiently small we have

$$
\int_{U}\|\bar{\nabla} h\|^{2} d x d y=\int_{U}|H|^{2} d v_{g}<\epsilon
$$

for any $\epsilon$. Hence

$$
\int_{\Sigma-U}|H|^{2} d v_{g}<(C+1) \epsilon+2 \epsilon^{1 / 2}
$$

for any $\epsilon$ and this shows $H \equiv 0$. 


\section{REFERENCES}

[BdC] J.L. Barbosa and M. doCarmo, On the Size of a Stable Minimal Surface in $\mathbf{R}^{3}$, Amer. J. Math 98(1976) 515-528. MR 54:1292

[B] R. Bryant, Minimal Lagrangian Submanifolds of Kähler-Einstein Manifolds, Lecture Notes in Math. 1255, 1-12, Springer-Verlag, New York, 1987. MR 88j:53061

[dCP] M. doCarmo and C.K. Peng, Stable Minimal Surfaces in $\mathbf{R}^{3}$ are planes, Bull. Amer. Math., 98 (1976) 515-528. MR 54:1292

[Ch] Q. Chen, On the Total Curvature and Area Growth of Minimal Surfaces in $\mathbf{R}^{n}$, Manu. Math., 92(1997) 135-142. MR 98c:49073

[C] S.S. Chern, Minimal Surfaces in an Euclidean Space of $N$ Dimensions, Differential and Combinational Topology, A symposium in honor of Marston Morse, Princeton University Press, Princeton, 1965, 187-198. MR 31:5156

[CO] S.S. Chern and R. Osserman, Complete Minimal Surfaces in Euclidean n-Space, J. Analyse Math. 19(1967) 15-34. MR 37:2103

[FS] D. Fischer-Colbrie and R. Schoen, The Structure of Complete Stable Minimal Surfaces in 3-manifolds of Non-negative Scalar Curvature, Comm. Pure Appl. Math 33(1980) 199-211. MR 81i:53044

[HL] R. Harvey and H. B. Lawson, Calibrated Geoemtries, Acta Math., 148(1982) 47-157. MR 85i:53058

[H] A. Huber, On Subharmonic Functions and Differential Geometry in the Large, Comm. Math. Helv., 32(1966), 105-136.

[Li] P. Li, Complete Surfaces of at Most Quadratic Area Growth, Comm. Math. Helv., 72(1997) 67-71. MR 98h:53057

[M] M. Micallef, Stable Minimal Surfaces in Euclidean Space, J. Diff. Geo, (1994), 57-84. MR 85e:53009

[Oh] Y.G.Oh, Second Variation and Stabilities of Minimal Lagrangian Submanifolds in Kähler Manifolds, Invent. Math., 101(1990), 501-519. MR 91f:58022

[O1] R. Osserman, Proof of a Conjecture of Nirenberg, Comm. Pure Appl. Math. 12(1959) 229-232. MR 21:4436

[O2] R. Osserman, On Complete Minimal Surfaces, Arch. Rational Mech. Anal., 13(1963) 392404. MR 27:1888

[O3] R. Osserman, Global Properties of Minimal Surfaces in $\mathbf{R}^{3}$ and $\mathbf{R}^{n}$. Ann. of Math. 2, 80(1964) 340-364. MR 31:3946

[SSY] R. Schoen, L. Simon and S.T.Yau, Curvature Estimates for Minimal Hypersurfaces, Acta Math., 134(1975) 275-288. MR 54:11243

[SW] R. Schoen and J Wolfson, Minimizing Volume Among Lagrangian Submanifolds. preprint. MR 99k:53130

[W] J. Wolfson, Minimal Lagrangian Diffeomeorphisms and the Monge-Ampére Equation, to appear J. Diff. Geo.

[X] F. Xavier, The Gauss Map of a Complete Non-flat Minimal Surfaces Cannot Omit 7 Points on the Sphere, Ann. of Math. 113(1981) 211-214.

Department of Mathematics DRB155, University of Southern California, 1042 West 36th Place, Los Angeles, California 90089

Current address: Box 5657, Department of Mathematics, Connecticut College, 270 Mohegan Ave., New London, Connecticut 06320

E-mail address: cmes@conncoll.edu 